\title{
Evaluation of Active Disease in a Patient of Non- Hodgkin Lymphoma by PET-CT scan over Non Significant CT, MRI Findings: a case report
}

\author{
Abu Bakker Siddique, Mohammad Simoon Salekin, Fatima Begum and Shamim M F Begum \\ National Institute of Nuclear Medicine and Allied Sciences \\ Correspondence Address : Dr. Abu Bakker Siddique, Associate Professor/PMO, PET-CT division, National Institute of Nuclear \\ Medicine \& Allied Sciences, BSMMU Campus, Shahbagh, Dhaka. E-mail: siddiquemukta2013@gmail.com
}

\begin{abstract}
Imaging modalities like computed tomography (CT) and MRI (magnetic resonance imaging) have been used as diagnostic tools for decades for screening as well as staging for any malignancies but these scans provide anatomical information only. After introduction of 18 Flurodeoxyglucose (FDG) positron emission tomography-computed tomography (PET-CT) hybrid imaging modality as molecular imaging technique by measuring glucose metabolism has revolutionized the detection capability of tumor. PET-CT scan has been used in localization and staging of lymphoma for two decades worldwide. Here, a case of 52 years male, diagnosed case of non-Hodgkin lymphoma (NHL) and received chemotherapy. At the end of therapy results of conventional imaging by CT, MRI suggested complete response of the disease. However, simultaneous PET-CT scan showed extensive active disease. PET-CT hybrid imaging provides additive information about location and extent of the active tumor over CT \& MRI, which have limitation to define functional lesion.
\end{abstract}

Key words: Lymphoma, PET-CT.

Bangladesh J. Nucl. Med. Vol. 21 No. 2 July 2018

Doi : https://doi.org/10.3329/bjnm.v21i2.40369

\section{INTRODUCTION}

Lymphomas are a group of hemopoietic disease arising from cells of immune system or form their precursors. Amongst the many subtypes of lymphomas, non Hodgkin lymphoma (NHL) is an aggressive variant. Continuous surveillance for evaluation of rapid staging and restaging is needed for planning of next step of treatment. Imaging modalities like computed tomography (CT) and MRI (magnetic resonance imaging) have been used as diagnostic tools for decades for screening as well as staging for any malignancies. However, due to some limitations sometimes CT or MRI alone fails to detect spread of any malignant tumor. Therefore it becomes difficult for accurate restaging as well as to determine the therapeutic response. Thus accurate level of metastasis or level of therapeutic response could not be measured by only anatomical imaging. The PET technology first introduced in 1973 with the first whole body PET in 1977, PET scan had been used as imaging modality since 1990, around the same time PET scans was first applied for assessment for lymphoma. Integrated PETCT as a hybrid imaging modality had been in regular patient's investigation workup since 2001 (1). Juweid et al were the first for assessment of lymphoma to incorporate FDG/PET into the then standard lymphoma response (2), because 18F FDG PET-CT measures regional glucose uptake, it is not only highly sensitive for metabolically active tissue but also in combination with a computerized (CT) scan for localization, spread $\&$ response evaluation. Though in patients with Hodgkin lymphoma, FDG PET-CT is highly sensitive for predicting therapeutic response, in case of non Hodgkin lymphoma it is less due primarily to because of heterogeneity and lack of evidence of reasonable predictive value (3). During the last two decades, patient imaging workup with 18F FDG PET-CT scan continued to increase, particularly in the field of nuclear oncology.

Here a case of NHL was presented who was considered as complete response to therapy on $\mathrm{CT}$ and 
MRI and referred to NINMAS for PET-CT scan for evaluation of disease state.

\section{CASE REPORT}

A 52 years, diabetic male with known case of situs inversus was diagnosed as NHL. Following diagnosis he was referred to oncologist and was prescribed with initial chemotherapeutic regimens. Following 6 cycles of chemotherapy oncologist tried to assess the treatment response \& restaged the patient using conventional imaging modalities like CT \& MRI. Hisis last chemotherapeutic cycle was uneventful. He underwent MRI of lumbosacral spine, that showed lumbar lordosis, Schmorls's node at end plate of body of 1 st sacral vertebra, early disc desiccation in between 4th and 5th lumbar vertebra and broad based diffuse disc bulge with traversing of 5 th lumbar nerve root. CT scan of neck with both pre $\&$ post contrast enhancement failed to detect any lymph node in neck (Figure 1).

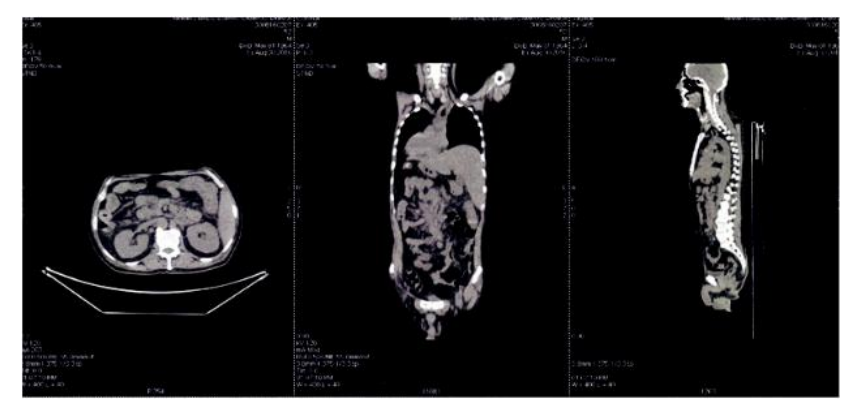

Figure 1: CT scan (from left to right: Axial, coronal \& sagittal view) of 52 years male with $\mathrm{NHL}$ after treatment showing no anatomical lesions corresponding PET scan. Situs inversus is seen in coronal view.

CT chest on same day suggest ill defined opacities or increased attenuation and striation at different segments of both lower lobes and right middle lobe; which showed compared with significant regression of pulmonary lesion and disappearance of mediastinal lymphadenopathy with absence of any pleural effusion. CT abdomen revealed small cortical cysts in both kidneys with sub centrimeter lymph nodes in abdomen suggesting remarkable regression of size and extension abdominal lymphadenopathy. Oncologist after reviewing all the results concluded that the patient showed complete response to chemotherapy (Figure 1). However, cerebro spinal fluid cytology revealed positive malignant cell; the oncologist re referred the patient to NINMAS for PET-CT evaluation. The patient underwent 18F FDG PET-CT scan following 6 hours of overnight fasting. After administration of $5 \mathrm{~m}$ Ci18F FDG the patient was kept in resting quietly for 60 minutes in a shielded room. Imaging was performed with GE-Discovery TM PET-CT series 710 with LYSO crystal scanner. Scan was performed from vertex to mid thigh with 9 bed positions, 2 minutes per bed. Fasting blood glucose level was $5.9 \mathrm{~m} \mathrm{~mol} / \mathrm{L}$ at the time of injection. Standardized Uptake Value (SUV) was calculated as SUV bwgm $/ \mathrm{ml}$. After careful evaluation following results were noted-extensive focal, linear \& diffuse FDG avid lesions (SUV max-13; most intense lesion) throughout the spine, ribs, sternum, scapulae, both hip bones, upper parts of both femora $\&$ upper end of right humerus suggested extensive bony meatstases (Figure 2, Figure 3).

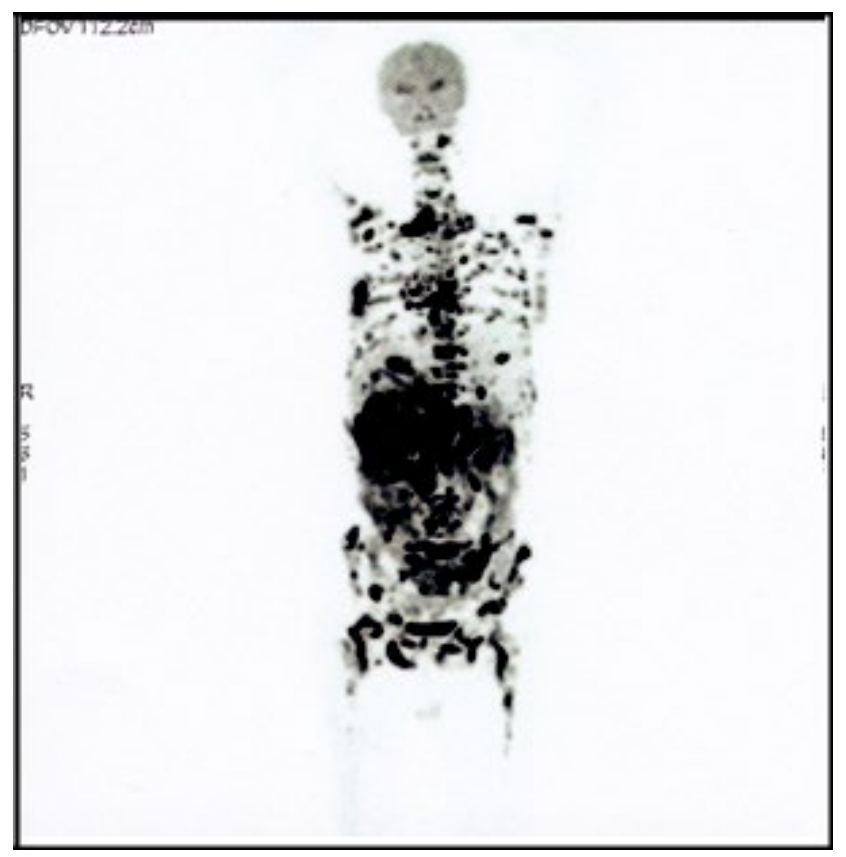

Figure 2: MIP (Maximum intensity projection) of PET-CT scan of 52 years male with NHL after treatment showing extensive FDG avid metastases. 
Multiple focal hypermetabolic (SUVmax-6.0) lesions are scattered in both lobes of liver. Focal FDG lesions were also noted in both sides of neck at level VB (SUVmax-8.0 on left \& SUVmax- 7.0 on right side). Multiple focal hypermetabolic (SUVmax -10 most intense) in both para tracheal, retro sternal, subcarinal, para esophageal, paraaortic regions in chest \&both inguinal (SUVmax - 12 in left side) and right internal iliac (SUVmax-21) clearly delineating extensive nodal metastases (Figure 3, Figure 4). Restaging of the patient was Staging IV of NHL.

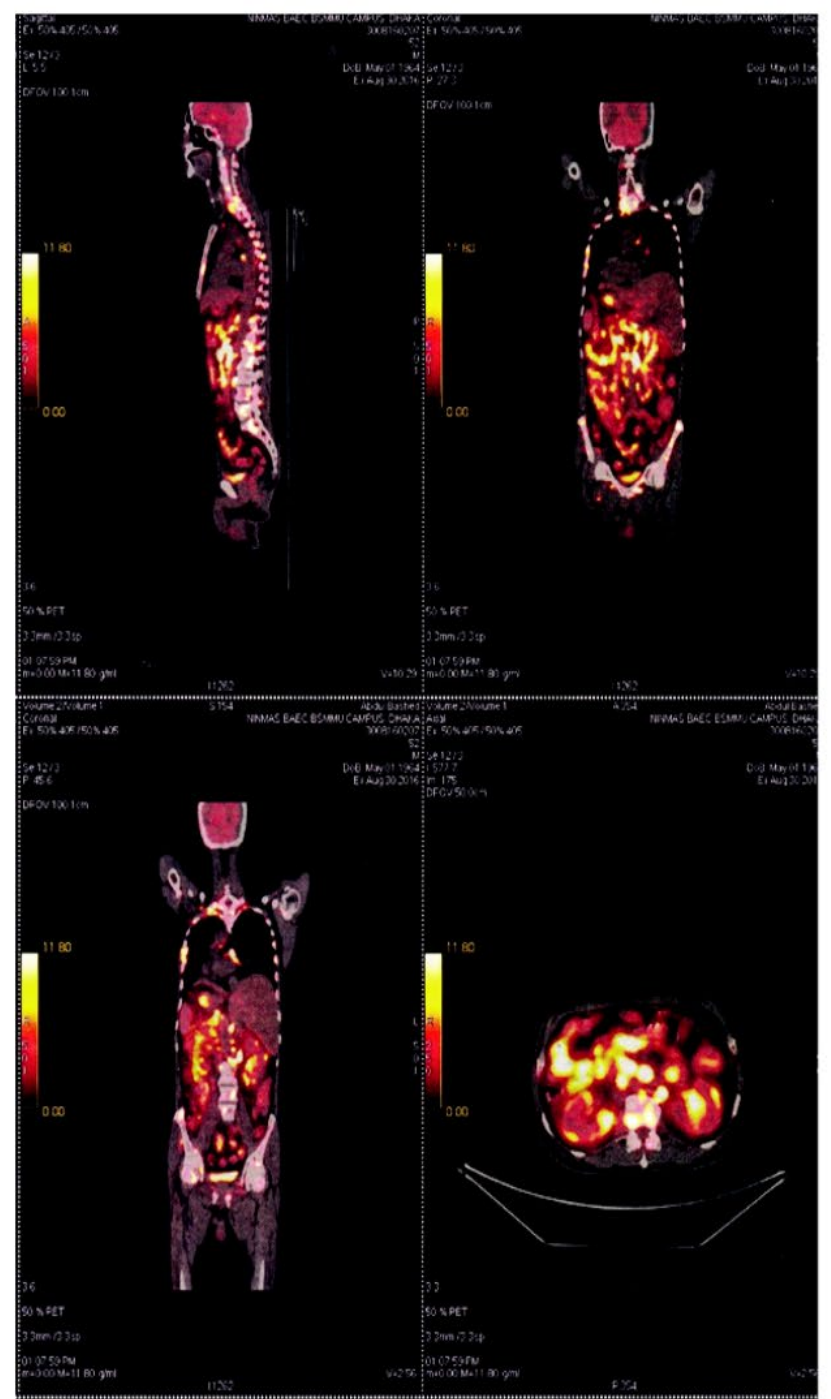

Figure 3: Fusion PET-CT scan (upper left: sagittal, upper right \& lower left: coronal, lower right: axial view)of a 52 years male with NHL after treatment showing extensive FDG avid metastases.

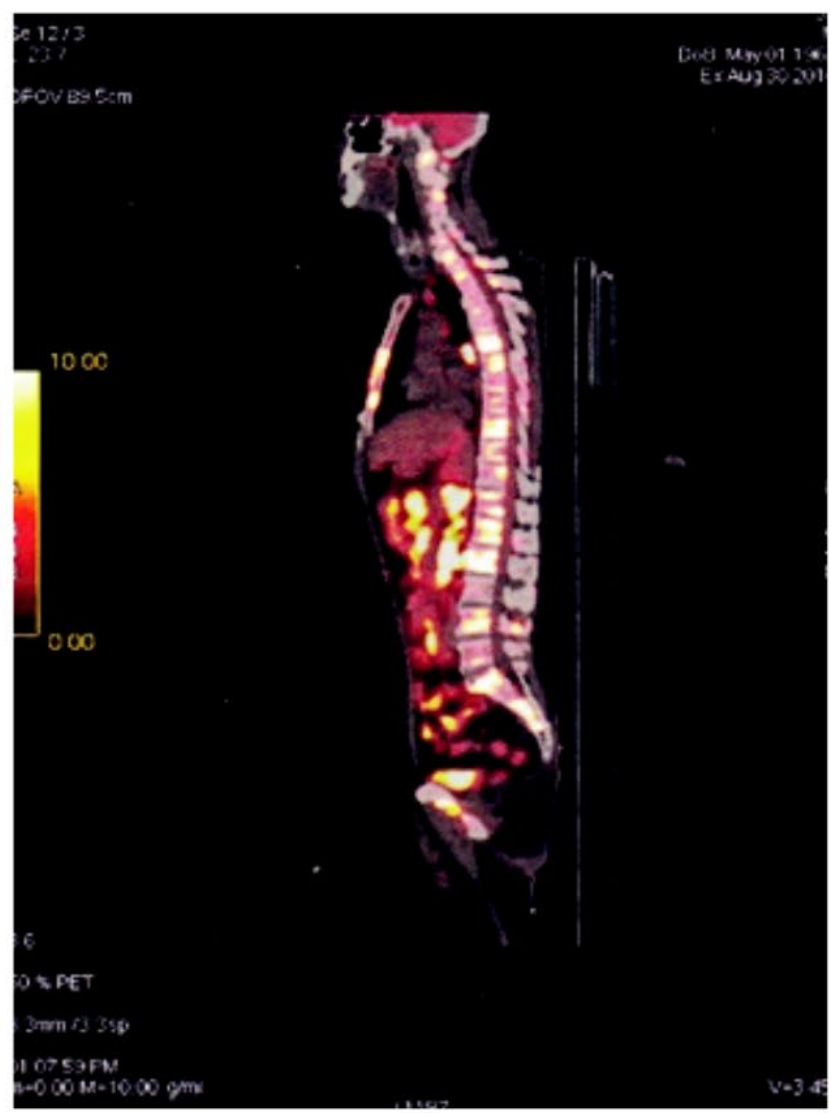

Figure 4: Fusion PET-CT (sagittal view) showing extensive bony metastases.

\section{DISCUSSION}

Lymphoma is one of most epidemic hemopoietic disease of immune cells. Due to its heterogeneity and many subtypes, staging is necessary to predict accurate therapeutic response. Conventional imaging techniques like computed tomography (CT) and magnetic resonance imaging (MRI) are originally routinely used as for staging of any lymphoma. However, due to inception of newer hybrid imaging PET-CT in developed world and recently at National Institute of Nuclear Medicine \& Allied Sciences (NINMAS) since 2016 at regular basis; the oncologists are referring for staging and restaging of lymphoma. Here a case of NHL was presented who was considered as complete response to therapy on CT and MRI and referred PET-CT scan for evaluation of disease state. 
18F-FDG, most common used PET radio tracer is transported to metabolically active cells via glucose transporter proteins and subsequently phosphorylated. Phosphorylated 18 F-FDG cannot typically dephosphoprylate and trap in the cell. Malignant tumours express higher numbers of specific membrane transport proteins, with greater affinity for glucose than normal cells, which permit increased glucose flow in the malignant cells. 18F FDG accumulates in tumor cells and positrons from $18 \mathrm{~F}$ generate two $511 \mathrm{KeV}$ photons in opposite direction, these eventually reconstructed into metabolically functional imaging of a tumor. Co-registration of low dose CT allows attenuation correction as well as anatomic information in this single setting (Figure-1, Figure-2) (4). 18F FDG avidity of lymphoma correlates better with histopathologic subtypes rather than clinical characteristics after incorporating of PET into the international working group criteria for lymphoma staging in 2007. PET - CT is now widely accepted as an imaging tool for evaluating end of treatment response in lymphoma. The ground work for the Deauville 5-point scale (D5PS) criteria is now expanded by Lugano guidelines, which established PET-CT as modality of choice for staging and treatment response assessment (5).

This particular patient who was previously diagnosed with non-Hodgkin lymphoma, underwent interim CT \& MRI imaging for finding out treatment response. Though repeated scan was done, CT \& MRI both failed to predict the lymph node involvement in abdomen. Sub centimeter lymph nodes in abdomen were diagnosed more as inflammatory and vertebral changes were suspected as degenerative disease. Though ill defined opacities in lungs were present, due to absence of pleural effusion along with hilar lymphadenopathy; infiltration was ruled out. Raanani et al found 31\% upstaging (6). 18F FDG PET-CT imaging modality has ruled out this false negative result of CT \& MRI disease regression to wide spread active disease in this case. In another study, PET-CT evaluated additional lesions in 30 patients out of 87 lymphoma over diagnostic CT patients, of which 11 patients showed increased clinical stage (7). According to international criteria for staging of lymphoma, overall extensive lymphadenopathy as well as bone marrow infiltration of lymphoma, the restaging for NHL of this patient was stage-IV.

\section{CONCLUSION}

18F FDG PET-CT played an important role for diagnosis of recurrence and restaging of lymphoma when CT \& MRI showed disease free state of this particular patient. In this case, the diagnostic value of 18F FDG PET-CT after end of therapy showed superiority than anatomical imaging modalities like $\mathrm{CT}$ and MRI.

\section{REFERENCES}

1. Bruce D. Cheson. PET/CT in Lymphoma: Current Overview \& Future Directions"; Seminars in Nuclear Medicine 2018;48:1. https://doi.org/10.1053/j.semnuclmed.2017.09.007.

2. Juweid M. Wiseman GA, Vose GM et al, “ Response assessment of non hodgkin's lymphoma by integrated international workshop criteria (IWC) and 18 FDG-PET. J Clin Oncol 2005;23: 4652-4661. PMID:15837965.

DOI:10.1200/JCO.2005.01.891

3. Deepa Bhojwani, Mary bethMcCarville, John K Choi, Jennifer Sawyer, Monika L. Metzger et al. The role of FDG-PET/CT in the evaluation of residual diseases in pediatric non-hodgkin lymphoma. Br J Haematol. 2015 March;168 (6): 845-853. doi: 10.1111/bjh.13219

4. Xuejuan Wang. PET-CT: appropriate application in lymphoma. Chin Clin Oncol 2015: 4(1): 4. Doi: 10.3978/j.jssn 23043865.2014.11.05.

5. Bruce D. Cheson, Richard I. Fisher, Sally F. Barrington, Franco Cavalli, Lawrence H. Schwartz, Emanuele Zucca, and T. Andrew Lister. Recommendations for initial evaluation, staging and response assessment of Hodgkin \& Non-hodgkin lymphoma;: the Lugano classification. J Clin Oncol 2014;32: 3059-3068. doi: 10.1200/JCO.2013.54.8800

6. Raanani P, Shasha Y, Perry C, et al: Is CT scan still necessary for staging in Hodgkin and non-Hodgkin lymphoma patients in the PET/CT era? Ann Oncol 2006;17:117-122. DOI:10.1093/annonc/mdj024

7. Elstrom R, Leonard JP, Coleman M, et al: Combined PET and low-dose, noncontrast CT scanning obviates the need for additional diagnostic contrast-enhanced CT scans in patients undergoing staging or restaging for lymphoma. Ann Oncol 2008;19: 1770-1773. DOI:10.1093/annonc/mdn282 